\section{Celiac CTLs change their stripes}

Gut-resident $\mathrm{T}$ cells morph into disease-causing natural killer (NK)like cells during Celiac disease (CD), according to Meresse and colleagues on page 1343 .

For people with CD, dietary gluten is the enemy. Gluten consumption somehow stresses the epithelial lining of the gut and triggers the activation of gluten-specific CD4 ${ }^{+} \mathrm{T}$ cells. $\mathrm{CD}^{+}$cytolytic $\mathrm{T}$ lymphocytes (CTLs) in the gut also expand during CD, but their role in disease is less clear.

Meresse and colleagues now show that gut CTLs from patients with CD express high levels of the activating NK cell receptor NKG2C, which is typically expressed only by NK cells. Binding of NKG2C by its receptor HLA-E (which was expressed on diseased gut epithelium) caused the CTLs to kill NK target cell lines, proliferate, and produce interferon (IFN)- $\gamma$-all in the absence of T cell receptor (TCR) ligation. In short, it caused them to act like NK cells.

This induction of NKG2C on gut CTLs might free them from their normal TCR-mediated activation constraints, thus unleashing them on gluten-stressed gut cells. NKG2C ${ }^{+}$CTLs from patients with CD showed that these cells_-but not normal CTLs - also expressed other activating NK cell receptors, suggesting that the cells get reprogrammed during CD.

Why would the immune system de-evolve antigen-restricted CTLs into indiscriminate killers? The authors speculate that this may have developed to help clear local infections, as NK cells have a hard time penetrating tissues, but that the process is taken to the extreme during CD. JEM

\section{Platelets pull in progenitors}

Clot-forming platelets lure vessel-rebuilding progenitor cells to sites of blood vessel injury, according to Massberg and colleagues on page 1221.

Injured blood vessels are repaired in part by bone marrow (BM)-derived progenitor cells, which migrate to the afflicted vessel and differentiate into new vascular cells. The chemokine SDF-1 attracts these cells and is reportedly produced by dying smooth muscle cells (SMCs). But the timing didn't add up. Production of SDF- 1 by SMCs requires at least six hours, whereas the recruitment of progenitor cells begins within minutes of vessel injury.

Massberg et al. now account for the short time lag by showing that platelets-not previously thought to release SDF-1-provide an early burst of the chemokine that helps reel in the first progenitor cells. In fact, although SMCs eventually produced SDF-1, blocking platelet adherence completely prevented the recruitment of progenitor cells.

Platelet adherence was strictly required because the progenitor cells lacked the receptors necessary to tether them directly to the injured vessel wall. Instead, they glommed onto the aggregated platelets, an interaction that required the binding of P-selectin on the platelet to its ligand PSGL-1 on the progenitor cell.

The group is now investigating the recruited progenitor cell fate, which has never been convincingly established. Depending on what the cells becomeeither endothelial cells or SMCs-they could either help rebuild injured vessels or contribute to a dangerous vessel wall thickening. JEM

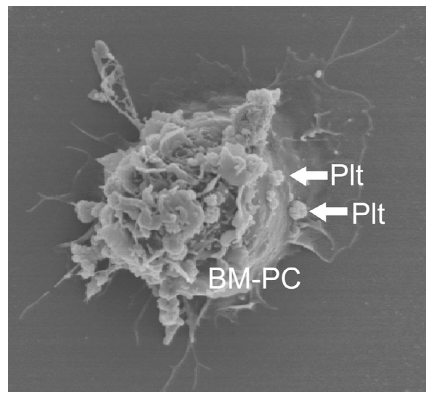

Bone marrow-derived progenitor cells (BM-PC) bind directly to platelets (PIt) at sites of blood vessel injury.

\section{Transforming with telomerase}

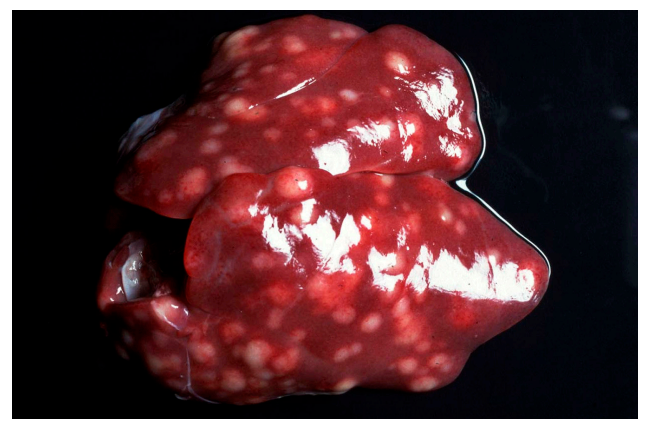

The telomerase RNA encoded by Marek's disease virus promotes tumor formation in chickens.

Viruses often transform their host cell so they can have a long-lasting and often mobile home. Now, Trapp et al. (page 1307) find that one chicken virus uses a hijacked telomerase RNA (TR) to trigger tumor formation.

Marek's disease virus (MDV)-a tumorcausing herpesvirus of chickens-costs the poultry industry an estimated $\$ 1$ billion annually. Although vaccines against MDV exist, the crowded living conditions of commercially raised chickens have fostered the evolution of nastier, vaccine-resistant virus strains.

The design of better vaccines, however, requires a better understanding of how the virus triggers tumors. Transformation is driven in part by the viral protein Meq, a Jun/Fos-like oncoprotein. The virus also encodes its own TR (vTR)-most likely pirated from the chicken genome.

TR is part of the telomerase enzyme that adds protective ends-telomeres-onto chromosomes. Telomerase activity is increased in many human tumors, but whether this is a cause or an effect of transformation has never been firmly established.

Trapp and colleagues now provide the first evidence that a vTR can contribute directly to tumor formation in a natural animal model. Wild-type chickens infected with MDV strains lacking both copies of the TR gene, they show, were less likely to develop cancer despite normal virus replication. The tumors that did develop were less widely disseminated.

The vTR does not appear to promote tumor formation by adding extra length to chromosome ends, as cells expressing vTR had normal-length telomeres. One clue might be the increased expression of $\alpha v$ integrins on vTRexpressing cells, as $\alpha v$ integrins have been implicated in promoting metastasis. JEM 